\title{
Macular Pigment Optical Density is Related to Blood Glutathione Levels in Healthy Individuals
}

\author{
Lu Qin, ${ }^{1,2}$ Hannab Bartlett, ${ }^{1,2}$ Helen R. Griffiths, ${ }^{2}$ Frank Eperjesi ${ }^{1,2}$ \\ Richard A. Armstrong, ${ }^{1,2}$ and Doina Gherghel ${ }^{1,2}$
}

Purpose. To assess the relationship between macular pigment optical density (MPOD) and blood markers for antioxidant defense in otherwise healthy volunteers.

Methods. Forty-seven healthy volunteers were subjected to blood analysis to detect the level of circulating glutathione in its reduced (GSH) and oxidized (GSSG) forms. The level of MPOD was measured using heterochromatic flicker photometry. Systemic blood pressure (BP) parameters, heart rate (HR), body mass index (BMI), and plasma levels of total, HDL, and LDL cholesterol and triglycerides (TGs) were also determined.

RESULTS. A simple correlation model revealed that the level of MPOD correlated significantly and positively with both GSH $(P<0.001)$ and $\mathrm{t}-\mathrm{GSH}(P<0.001)$ levels but not with those of GSSG $(P>0.05)$. Age, sex, systemic BP parameters, HR, BMI, and plasma levels of cholesterol and TGs did not have any influence on either MPOD or glutathione levels (all $P>0.05$ ). In addition, a forward stepwise multiple regression analysis showed MPOD to have a significantly and independent correlation with GSH levels $(\beta=0.63 ; P<0.001)$

Conclusions. In otherwise healthy older individuals, there is a positive correlation between local and systemic antioxidant defense mechanisms. (Invest Ophthalmol Vis Sci. 2011;52: 5029-5033) DOI:10.1167/iovs.11-7240

mong environmental, nutritional, and genetic risk factors
involved in the etiology of AMD, high levels of oxidative
stress, a harmful state defined by the presence of pathologic
levels of reactive oxygen species (ROS) relative to the antiox-
idant defense, have been said to play a role. ${ }^{1-3}$ In healthy
subjects, local retinal protection against free radicals is aided
by the presence of macular pigment (MP), which is comprised
of the carotenoid lutein and its isomers zeaxanthin ${ }^{4}$ and meso-
zeaxanthin. ${ }^{5}$ It has been suggested that these xanthophylls play
a similar role in humans as in plants- that is, as antioxidants
and screeners of high-energy blue light. ${ }^{6}$ In this way, MP may
prevent light-initiated oxidative damage to the retina and there-
fore protect against subsequent AMD. ${ }^{7}$ Indeed, the absorbance
spectrum of MP peaks at 460 nm and it is thought to act as a
broadband filter, reducing the sensitivity of the macular region
to short wavelength light, which is most damaging in the 440
to 460 nm range. ${ }^{8,9}$ In addition, the fact that lutein and zeax-

From the ${ }^{1}$ Ophthalmic Research Group and the ${ }^{2}$ Aston Research Centre for Healthy Ageing, School of Life and Health Sciences, Aston University, Birmingham, United Kingdom.

Submitted for publication January 17, 2011; revised April 6, 2011 and April 27, 2011; accepted April 27, 2011

Disclosures: L. Qin, None; H. Bartlett, None; H. R. Griffiths None; F. Eperjesi, None; R. A. Armstrong, None; D. Gherghel, None.

Corresponding author: Doina Gherghel, School of Life and Health Sciences, Aston University, Aston Triangle, Birmingham, B4 7ET, United Kingdom; d.gherghel@aston.ac.uk. anthin have been found in higher concentrations in the rod outer segments of the perifoveal retina than the peripheral retina lends support to their proposed protective role against AMD ${ }^{10}$ These carotenoids are able to quench singlet oxygen (a potent oxidant),${ }^{11}$ scavenge ROS,${ }^{12}$ limit the peroxidation of membrane phospholipids, ${ }^{13}$ and reduce lipofuscin formation. $^{14}$

In addition to local retinal damage, high levels of oxidative stress also induces vascular changes that confer a background for circulatory disturbances in the systemic macro- and microcirculation that are also present in AMD patients. ${ }^{15,16}$ Indeed, it has been shown that in addition to ocular vascular complications, AMD patients are at higher risk for developing coronary heart disease (CHD) and stroke ${ }^{17,18}$; moreover, they also are more likely to have a decreased survival rate compared to the general age-matched population. ${ }^{19}$ It has been shown that AMD patients have lower levels of circulating glutathione (GSH), a major low-molecular weight antioxidant peptide ${ }^{20}$; in addition to a direct vascular effect, this deficiency could also result in low bioavailability of the vasodilatory molecule nitric oxide $(\mathrm{NO})^{21}$ and endothelial dysfunction, a condition known to precede both metabolic and cardiovascular diseases but also ocular circulatory disturbances.

It has been reported that dietary intake of lutein and zeaxanthin augument the level of MP with a possible positive effect on AMD prevention and prognosis. ${ }^{22,23}$ In addition, carotenoid-rich food intake has a positive influence on systemic antioxidant status in healthy persons. ${ }^{24}$ The aim of the present study was to determine whether there is a relationship between local and systemic antioxidant defense markers in older volunteers free of either ocular or systemic diseases.

\section{Materials ANd Methods}

\section{Study Sample}

Healthy subjects recruited by advertising at Aston University, Birmingham, United Kingdom were considered for inclusion in this prospective study. Ethical approval was sought from the local ethics committee, and written informed consent was obtained from all participants before enrolling into the study. The study was designed and conducted according to the principles of the Declaration of Helsinki.

Exclusion criteria were smoking, a history of any chronic systemic disease with presumed abnormal circulating GSH levels, including autoimmune diseases ${ }^{25}$ alcoholic liver disease, ${ }^{26}$ cancer,${ }^{27}$ and diabetes mellitus. ${ }^{28}$ In addition, subjects were also excluded if they had cardiovascular or cerebrovascular disease, coronary artery disease (heart failure, arrhythmia, stroke, or transient ischemic attacks), inflammatory conditions (rheumatoid arthritis and systemic lupus erythematosus), or were receiving hormone replacement therapy or antioxidant/vitamin/iron supplements.

Potential participants were screened for ocular diseases by an ophthalmologist and patients with ocular diseases, such as cataracts, glaucoma, and AMD, were also excluded from the study. Retinal 
photographs were taken using a digital camera (Zeiss FF 450 Plus Fundus Camera; Carl Zeiss Meditec, Inc, Jena, Germany) and the presence or absence of drusen or pigmentary changes associated with AMD was assessed before inclusion in the study. In addition, potential participants were required to complete a questionnaire on their general health, physical activity, and alcohol consumption. The questionnaire included a detailed investigation on the daily intake of fruit and vegetables and other nutrients.

\section{Investigations}

Subjects were instructed to fast after $9 \mathrm{PM}$ on the evenings before being tested. On the morning of the test, subjects were requested to have only a light breakfast, such as toast. They were also asked to avoid any cooked breakfast, meat, cereal, fresh fruits, or fruit juice. ${ }^{29}$ In addition, subjects were asked to abstain from caffeinated beverages and chocolate and from alcohol for at least 2 hours before the visit. The height and weight of the participants was measured and the body mass index (BMI) was calculated (as $\mathrm{kg} / \mathrm{m}^{2}$ ).

\section{Blood Pressure Measurement}

Blood pressure (BP) was measured in each subject in the morning between $8 \mathrm{AM}$ and $9 \mathrm{AM}$ with a $\mathrm{BP}$ monitor (UA-779; A7D Instruments Ltd., Oxford, UK). In preparation for this measurement, each subject rested in a sitting position for approximately 10 minutes in a quiet room to achieve sufficient mental and physical calm. The systolic BP (SBP) and diastolic BP (DBP) were measured three times (1 minute apart). The average readings for SBP and DBP were then used to calculate the mean $\mathrm{BP}(\mathrm{MBP})$ using the formula: $\mathrm{MBP}=2 / 3 \mathrm{DBP}+$ $1 / 3$ SBP.

\section{Blood Sampling and Analyses}

All blood samples were obtained by a qualified phlebotomist between $9 \mathrm{AM}$ and $10 \mathrm{AM}$.

\section{Routine Tests}

Fasting, triglycerides (TG), and total and high-density lipoprotein (HDL) cholesterol levels were measured automatically using a Reflotron Desktop Analyzer (Roche Diagnostics, Welwyn Garden City, UK).

\section{Blood Sampling for GSH Analyses}

Seven milliliters of blood was collected in EDTA tubes (to prevent oxidation $)^{30}$ by venipuncture to the antecubital vein using a butterfly needle and syringe to avoid hemolysis. ${ }^{20}$ The blood $(30 \mu \mathrm{L})$ was then transferred into centrifuge tubes for the initial processing. GSH was released from the blood cells by protein precipitation and cellular disruption achieved by addition of $33.3 \mu \mathrm{L}$ of 5 -sulfosalicylic acid (SSA), $1 \mathrm{~g} / \mathrm{mL}$ within 10 minutes from the blood collection. ${ }^{31}$ The addition of acid minimizes artifactual sample oxidation and removes interfering protein thiols.

Each sample was then diluted with $936.7 \mu \mathrm{L}$ sodium phosphate buffer $(\mathrm{pH} 7.5)$ and the content of each tube was rapidly centrifuged at $13,000 \mathrm{rpm}$ for 5 minutes. Small amounts $(150 \mu \mathrm{L})$ of supernatant were collected into clean centrifuge tubes and immediately cooled at $-70^{\circ} \mathrm{C}$. Samples stored at this temperature are stable for at least 2 months and can be transported on dry ice without deterioration. ${ }^{32}$ In our hands, GSH loss is $<5 \%$ over this time period.

\section{GSH Assay}

The total GSH levels (t-GSH) were assessed by the GSH reductaseDTNB (5.5 dithiobis-2-nitrobenzoic acid) recycling procedure, as described in previous studies. ${ }^{30,33,34}$ Standards were prepared from 0 to $80 \mu \mathrm{M}$ in $20-\mu \mathrm{M}$ increments using $10 \mathrm{mM}$ GSH solution. The standards contained the same final concentrations of SSA as the samples. To each well of a 96-well plate, NADPH $(0.3 \mathrm{mg} / \mathrm{mL})$, dissolved in $150 \mu \mathrm{L}$ of 125 $\mathrm{mM}$ sodium phosphate with $6.3 \mathrm{mM}$ EDTA $\mathrm{pH} 7.5$, also known as daily buffer, $50 \mu \mathrm{L}$ of DTNB solution, and $25 \mu \mathrm{L}$ of standards or samples were added in quadruplicate and the plate was incubated at $37^{\circ} \mathrm{C}$ for 3 minutes. Finally, $25 \mu \mathrm{L}$ of GSH reductase (GSR) was added to the previous mixture and the plate was read at $410 \mathrm{~nm}$ using a 96-well plate reader. A standard curve of GSH was then generated using a linear regression program (Microsoft Excel; Microsoft, Redmond, WA) as previously reported. ${ }^{34}$

\section{GSSG Assay}

GSSG levels were assessed using a GSH reductase-DTNB recycling assay. ${ }^{30}$ The reagents used in this assay were those already described above for GSH assay and, in addition, triethanolamine (TEA) and 2-vinyl pyridine (2-VP). TEA prevents a high local $\mathrm{pH}$ and oxidation, while 2-VP is used for derivitization of GSH. GSSG standards were prepared from 0 to $10 \mu \mathrm{M}$ in $1-\mu \mathrm{M}$ increments; $100 \mu \mathrm{L}$ of standards and samples were transferred into separate centrifuge tubes and $2 \mu \mathrm{L}$ 2-VP was added to each tube. TEA was then used to adjust the $\mathrm{pH}$ of the standards/samples to $\mathrm{pH} 7.5$. The assay was carried out as for GSH assay described above. Finally, a standard curve of GSSG was then generated using a linear regression program (Microsoft Excel) as previously reported. ${ }^{34}$

The GSH levels [t-GSH - $(2 \times$ GSSG)] and the redox index (defined as the GSH/GSSG ratio) were then calculated.

\section{Macular Pigment Optical Density Measurement}

Macular pigment optical density (MPOD) was determined using the MPS 9000 (also known as the M:Pod and the QuantifEYE; Topcon House, Berkshire, UK). This device measures MPOD using heterochromatic flicker photometry, in which subjects respond to the appearance of flicker as the alternation rate is decreased at $6 \mathrm{~Hz}$ per sec from a starting level of $60 \mathrm{~Hz} .{ }^{35}$ Because this is above the critical flicker fusion frequency for the test conditions, subjects do not perceive any flicker initially. A sequence of blue-green ratios is used and these are inverseyoked to ensure that overall luminance remains constant. The device determines each observer's sensitivity to flicker before the main part of the test. This technique is well documented, ${ }^{36}$ and the reliability of this particular instrument has also been assessed by our group. ${ }^{37}$

The eye not being tested was occluded and participants wore their habitual refractive correction (a trial frame and lenses were used when necessary). The central target is a $1^{\circ}$ circular stimulus composed of blue $(465 \mathrm{~nm})$ and green $(530 \mathrm{~nm})$ LEDs. For the foveal (central) test, the observer looked directly at the stimulus while the alternation rate between the blue and green was ramped down from $60 \mathrm{~Hz}$. At the point when they first detected flicker, the observer pressed a response button and this plotted a point on a graph that was visible to the operator on a computer screen. Once the flicker had been perceived, the process started again. The first five responses were used to ascertain the flicker sensitivity of the subject. The observer is asked to respond to a series of green-blue ratios until a V-shaped curve is plotted on the computer screen. The minimum point on the curve corresponds to equiluminance of the blue and green lights. The process was then repeated for the peripheral test, where the subject's gaze was directed to a larger red target, $8^{\circ}$ eccentric from the central spot. The difference between the central and peripheral minima is used by an internal algorithm to calculate the MPOD. Each subject was given a practice run of the test, and the results were screened such that if an appropriate V-shaped curve was not obtained, the test was repeated.

\section{Statistical Analysis}

Statistical analysis was performed using Statistica for Windows (version 9.0; StatSoft Inc., Tulsa, OK). Data are expressed as mean \pm SD. Pearson's correlation test and a forward stepwise multiple regression analysis were performed to test the relationship between the measured variables. $P$ values $<0.05$ were considered statistically significant.

\section{Results}

Sixty-one healthy subjects with similar dietary habits were selected for inclusion. However, after the evaluation of the 
TABLE 1. Characteristics of the Study Participants

\begin{tabular}{|c|c|c|c|c|c|c|c|c|}
\hline $\begin{array}{l}\text { Age, } y \\
( \pm \text { SD })\end{array}$ & Sex, F:M & $\begin{array}{l}\text { SBP, mm Hg } \\
\quad( \pm \text { SD })\end{array}$ & $\begin{array}{c}\text { DBP, mm Hg } \\
\quad( \pm \text { SD })\end{array}$ & $\begin{array}{l}\text { BMI, } \mathbf{~ k g} / \mathrm{m}^{2} \\
\quad( \pm \mathrm{SD})\end{array}$ & $\begin{array}{c}\text { Total } \\
\text { Cholesterol, } \\
\text { mmol/L }( \pm \text { SD })\end{array}$ & $\begin{array}{c}\text { HDL } \\
\text { Cholesterol, } \\
\text { mmol/L }( \pm \text { SD })\end{array}$ & $\begin{array}{c}\text { LDL } \\
\text { Cholesterol, } \\
\text { mmol/L }( \pm \text { SD })\end{array}$ & $\begin{array}{l}\text { TG, } \mathbf{m m o l} / \mathbf{L} \\
\quad( \pm \mathrm{SD})\end{array}$ \\
\hline $50 \pm 9$ & $29: 18$ & $118 \pm 13$ & $73 \pm 9$ & $25.20 \pm 4.15$ & $4.46 \pm 0.82$ & $1.29 \pm 0.33$ & $2.61 \pm 0.80$ & 0.62 \\
\hline
\end{tabular}

BMI, body mass index; DBP, diastolic blood pressure; F, female; HDL, high-density lipoprotein; LDL, low-density lipoprotein; M, male; SBP, systolic blood pressure; TG, triglycerides.

fundus photographs and eliminating those with potential macular changes, only 47 healthy subjects ( 29 women and 18 men) were included in the final analysis. The characteristics of the study participants are provided in Table 1 . Table 2 shows the anthropometric and vascular parameters determined by sex. Men exhibited higher SBP $(P=0.018)$ and DBP $(P=0.01)$ and lower levels of HDL cholesterol $(P=0.002)$ than women. However, MPOD and systemic oxidative markers were similar between the sexes (all $P>0.05$ ).

A simple correlation model revealed that the level of MPOD correlated significantly and positively with both blood GSH $(r=0.64 ; P<0.001)$ and $\mathrm{t}-\mathrm{GSH}(r=0.63 ; P<0.001)$ levels but not with those of GSSG $(P>0.05)$. Age, systemic BP parameters, BMI, and plasma levels of cholesterol and TGs did not have any influence on either MPOD or blood GSH levels (all $P>0.05$ ). A stepwise multiple regression analysis revealed MPOD levels to be independently, significantly, and positively correlated with blood GSH levels $(\beta=0.64 ; P<0.001$; Fig. 1$)$.

\section{Discussion}

At the retinal level, oxidative stress results in degeneration and death of the RPE and photoreceptors. ${ }^{38}$ Because these retinal structures are not able to regenerate after such insult, protective mechanisms have developed to ensure a minimal local effect of free radicals. ${ }^{39}$ Indeed, MP is present in the rod outer segments, and RPE and its specific spectral absorption and the presence of lutein and zeaxanthin have enabled it with strong, protective antioxidant properties. There are several methods for measuring the level of MP, including various subjective

TABLE 2. Measured Parameters for Men and Women

\begin{tabular}{|c|c|c|c|}
\hline & Female & Male & $\boldsymbol{P}$ \\
\hline Age, y $( \pm S D)$ & $49 \pm 9$ & $50 \pm 8$ & $>0.05$ \\
\hline MPOD ( \pm SD $)$ & $0.46 \pm 0.18$ & $0.47 \pm 0.12$ & $>0.05$ \\
\hline $\mathrm{TG}, \mathrm{mmol} / \mathrm{L}( \pm \mathrm{SD})$ & $1.16 \pm 0.65$ & $1.35 \pm 0.58$ & $>0.05$ \\
\hline $\begin{array}{l}\text { Total cholesterol, } \mathrm{mmol} / \mathrm{L} \\
\quad( \pm \mathrm{SD})\end{array}$ & $4.52 \pm 0.69$ & $4.37 \pm 1.00$ & $>0.05$ \\
\hline $\begin{array}{l}\text { HDL cholesterol, } \mathrm{mmol} / \mathrm{L} \\
\quad( \pm \mathrm{SD})\end{array}$ & $1.40 \pm 0.29$ & $1.11 \pm 0.30$ & 0.002 \\
\hline $\begin{array}{l}\text { LDL cholesterol, } \mathrm{mmol} / \mathrm{L} \\
\quad( \pm \mathrm{SD})\end{array}$ & $2.58 \pm 0.75$ & $2.65 \pm 0.89$ & $>0.05$ \\
\hline BMI, $\mathrm{kg} / \mathrm{m}^{2}( \pm \mathrm{SD})$ & $24.48 \pm 4.06$ & $26.37 \pm 4.13$ & $>0.05$ \\
\hline $\mathrm{SBP}, \mathrm{mm} \mathrm{Hg}( \pm \mathrm{SD})$ & $115 \pm 12$ & $124 \pm 12$ & 0.018 \\
\hline $\mathrm{DBP}, \mathrm{mm} \mathrm{Hg}( \pm \mathrm{SD})$ & $70 \pm 9$ & $77 \pm 8$ & 0.010 \\
\hline $\mathrm{HR}, \mathrm{bpm}( \pm \mathrm{SD})$ & $72 \pm 7$ & $69 \pm 8$ & $>0.05$ \\
\hline $\mathrm{t}-\mathrm{GSH}, \mu \mathrm{mol} / \mathrm{L}( \pm \mathrm{SD})$ & $1177 \pm 564$ & $1089 \pm 416$ & $>0.05$ \\
\hline $\mathrm{GSSG}, \mu \mathrm{mol} / \mathrm{L}( \pm \mathrm{SD})$ & $88 \pm 44$ & $78 \pm 51$ & $>0.05$ \\
\hline $\mathrm{GSH}, \mu \mathrm{mol} / \mathrm{L}( \pm \mathrm{SD})$ & $1002 \pm 526$ & $932 \pm 383$ & $>0.05$ \\
\hline $\begin{array}{l}\text { Redox index, GSH:GSSG } \\
\quad( \pm \text { SD })\end{array}$ & $11 \pm 7$ & $12 \pm 6$ & $>0.05$ \\
\hline
\end{tabular}

BMI, body mass index; DBP, diastolic blood pressure; GSH, reduced glutathione; GSSG, oxidized glutathione; HDL, high-density lipoprotein; HR, heart rate; LDL, low-density lipoprotein; MPOD, macular pigment optical density; SBP, systolic blood pressure; TG, triglycerides; t-GSH, total GSH. psychophysical and objective optical techniques. ${ }^{39}$ One of these subjective methods is represented by the heterochromatic flicker photometry that involves the calculation of MPOD based on the luminance ratio of short wavelength blue light presented in the central retina (where is assumed to be partially absorbed by the MP) compared to that presented at a more peripheral retinal point, where MP levels are assumed to be minimal. ${ }^{40}$ This method offers a good measure of the MPOD levels and is widely available in practice. By using this method, our analysis has shown for the first time an independent, significant, and positive relationship between MPOD and blood GSH levels. At this stage, more research is necessary to provide better knowledge of the exact mechanisms responsible; nevertheless, we can still propose a few hypotheses. It has been reported that the dietary intake of carotenoids had an influence not only on the level of $\mathrm{MP}^{22,23}$ but also on the systemic circulating antioxidant markers. ${ }^{24}$ In addition, exogenous supply of GSH protects the RPE against oxidative damage. ${ }^{41} \mathrm{Al}$ though the individuals included in the present study did not receive either a special diet rich in carotenoids nor GSH or other antioxidant supplementation, the aforementioned results simply show that a relationship between local and systemic protective mechanisms - such as that found by our studycould exist. Consequently, although novel, our results should not be surprising.

Other mechanisms can also be speculated. Melatonin, a neurohormone that is secreted by retinas and the pineal gland, has an influence on the RPE and controls the amount of light reaching the photoreceptors; in addition, it also acts as potent antioxidant at both the ocular and systemic level and, in such capacity, it has been advocated to reduce the risk for pathologies associated with high oxidative damage, such as $\mathrm{AMD}^{42}$ and cardiovascular disease. ${ }^{43}$ In addition, melatonin also activates other antioxidant defenses including GSH peroxidase (GPx), an enzyme that uses GSH as a substrate to eliminate ROS. ${ }^{44,45}$ Melatonin production could be affected by aging as the pupil diameter and the light absorption through the crystalline lens changes with age progression. ${ }^{46-48}$ Consequently, aging contributes to an abnormal melatonin production and

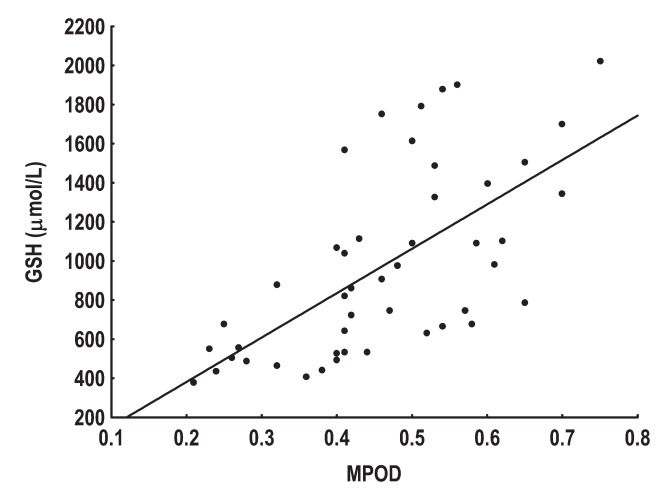

Figure 1. The correlation between the levels of macular pigment optical density and reduced glutathione in the study population $(P<$ $0.001)$. 
also decreases antioxidant defense, which in turn will result in accelerated aging processes with various effects throughout the human body, including the eye. Although measuring melatonin levels was not an aim in the present research, we could still hypothesize that a link between ocular and systemic antioxidant mechanisms could also be established indirectly via melatonin. This hypothesis should, however, be tested in various age groups. In addition, other mechanisms are most certainly involved and should be further researched using more complex analyses. The role of antioxidants proven to have a link to both macular pigment and circulating GSH levels should also be researched. Nevertheless, as previously emphasized, the positive correlation between the levels of MPOD and GSH seen in our study probably only reveal that, in healthy individuals, the antioxidant defense mechanisms present at various levels act in the same direction to protect the body against harmful effects of ROS. However-and maybe most importantlyour research points toward the necessity of studying various normal relationships between ocular and systemic protective mechanisms against diseases with multiple etiologies and complications, such as AMD. In this way, we could understand better results that are reported after various pathologic changes have already occurred. Reducing the risk for AMD is important, and strengthening natural bodily mechanisms that are at their best in healthy individuals seems to be one of the possible approaches.

Although some studies report sex differences in plasma GSH levels, ${ }^{49}$ others did not confirm it in either plasma or blood GSH. ${ }^{50}$ In agreement with later studies, we also could not find any difference between men and women with respect to blood GSH levels. It is possible that the various methods used for GSH assay are responsible for this lack of consistency in the results. There is no general agreement as to what method is best for analyzing circulating GSH. In the present study, we used a validated method for measuring blood levels of GSH and $\mathrm{GSSG}^{30,33,34}$ that is known to minimize variability from more complicated sample preparation steps associated with methods measuring plasma GSH. ${ }^{50}$ In addition, to avoid variations and GSH loss, we have paid particular attention to blood collection, initial processing, and storage. Blood samples were collected at the same time (between $9 \mathrm{AM}$ and $10 \mathrm{AM}$ ) and processed in the same way and time interval from collection in all individuals. Moreover, incorporation of GSR in our assay confers it specificity to GSH.

\section{Study Limitations}

As previously emphasized, our research included only patients over 40 years of age that were carefully selected to exclude macular changes or other ocular pathologies as well as systemic chronic disease and various therapeutic interventions. We have also excluded smokers and individuals taking any antioxidant supplements. This careful selection has limited the number of individuals included in the final analysis. It is possible that the relationship between MPOD and circulating GSH level is different not only in various age groups but also in individuals with additional risk factors for AMD and/or vascular disease. Moreover, individuals having a diet either lacking or very rich in carotenoids or receiving various supplements as well as patients suffering from AMD with or without vascular complications may display very different results. More studies to include larger and more various populations to cover the many possible confounders that have been either missed or intentionally avoided in the present study are warranted.

In conclusion, our study has shown for the first time that in older, healthy, nonsmoking individuals, MPOD correlates with whole blood levels of GSH. Because assays for measuring systemic levels of antioxidant molecules are complex and need specialized laboratories, it is tempting to propose that a simple MPOD assessment could be used as surrogate indicator of the individual's systemic capacity of dealing with the damaging effect of free radicals and, consequently, of their risk for developing chronic ocular and/or systemic pathologies. Nevertheless, this assumption should be verified in larger studies, and the clinical significance of associations such as the one reported by our research should be carefully analyzed.

\section{References}

1. Beatty S, Koh H, Phil M, Henson D, Boulton M. The role of oxidative stress in the pathogenesis of age-related macular degeneration. Surv Opbthalmol. 2000;45:115-134.

2. Cai J, Nelson KC, Wu M, Sternberg Jr P, Jones DP. Oxidative damage and protection of the RPE. Prog Retin Eye Res. 2000;19: 205-221.

3. Khandhadia S, Lotery A. Oxidation and age-related macular degeneration: insights from molecular biology. Expert Rev $\mathrm{Mol}$ Med. 2010;12:e34

4. Bone RA, Landrum JT, Tarsis SL. Preliminary identification of the human macular pigment. Vision Res. 1985;25:1531-1535.

5. Khachik F, de Moura FF, Zhao DY, Aebischer CP, Bernstein PS. Transformations of selected carotenoids in plasma, liver, and ocular tissues of humans and in nonprimate animal models. Invest Ophthalmol Vis Sci. 2002;43:3383-3392.

6. Krinsky NI. Possible biologic mechanisms for a protective role of xanthophylls. $J$ Nutr. 2002;132:540S-542S.

7. Hammond Jr BR, Wooten BR, Snodderly DM. Preservation of visual sensitivity of older subjects: association with macular pigment density. Invest Ophthalmol Vis Sci. 1998;39:397-406.

8. Reading VM, Weale RA. Macular pigment and chromatic aberration. J Opt Soc Am. 1974;64:231-234.

9. Pease PL, Adams AJ, Nuccio E. Optical density of human macular pigment. Vision Res. 1987;27:705-710.

10. Rapp LM, Maple SS, Choi JH. Lutein and zeaxanthin concentrations in rod outer segment membranes from perifoveal and peripheral human retina. Invest Ophthalmol Vis Sci. 2000;41:1200 1209.

11. Krinsky NI. Carotenoid protection against oxidation. Pure Appl Chem. 1979;51:649-660.

12. Di Mascio $P$, Kaiser S, Sies H. Lycopene as the most efficient biological carotenoid singlet oxygen quencher. Arch Biochem Biophys. 1989;274:532-538.

13. Lim BP, Nagao A, Terao J, Tanaka K, Suzuki T, Takama K. Antioxidant activity of xanthophylls on peroxyl radical-mediated phospholipid peroxidation. Biochim Biopbys Acta. 1992;1126: 178-184

14. Sundelin SP, Nilsson SE. Lipofuscin-formation in retinal pigment epithelial cells is reduced by antioxidants. Free Radic Biol Med. 2001;31:217-225.

15. Lip PL, Blann AD, Hope-Ross M, Gibson JM, Lip GY. Age-related macular degeneration is associated with increased vascular endothelial growth factor, hemorheology and endothelial dysfunction. Opbthalmology. 2001;108:705-710.

16. Zaliuniene D, Jasinskas V, Jurkevicius R, Gustiene O, Zaliunas R. Endothelial function, intima-media thickness, and ankle-brachial index in patients with cataract and age-related macular degeneration. Eur J Opbthalmol. 2008;18:384-391.

17. Wong TY, Klein R, Sun C, et al. Age-related macular degeneration and risk for stroke. Ann Intern Med. 2006;145:98-106.

18. Wong TY, Tikellis G, Sun C, Klein R, Couper DJ, Sharrett AR. Age-related macular degeneration and risk of coronary heart disease: the Atherosclerosis Risk in Communities Study. Opbthalmology. 2007;114:86-91.

19. Hyman L, Schachat AP, He Q, Leske MC. Hypertension, cardiovascular disease, and age-related macular degeneration. Age-Related Macular Degeneration Risk Factors Study Group. Arch $O p h$ thalmol. 2000;118:351-358.

20. Samiec PS, Drews-Botsch C, Flagg EW, et al. Glutathione in human plasma: decline in association with aging, age-related macular 
degeneration, and diabetes. Free Radic Biol Med. 1998;24:699704 .

21. Evereklioglu C, Er H, Doganay S, et al. Nitric oxide and lipid peroxidation are increased and associated with decreased antioxidant enzyme activities in patients with age-related macular degeneration. Doc Ophthalmol. 2003;106:129-136.

22. Wenzel AJ, Sheehan JP, Burke JD, Lefsrud MG, Curran-Celentano J. Dietary intake and serum concentrations of lutein and zeaxanthin, but not macular pigment optical density, are related in spouses. Nutr Res. 2007;27:462-469.

23. Bhosale P, Zhao da Y, Bernstein PS. HPLC measurement of ocular carotenoid levels in human donor eyes in the lutein supplementation era. Invest Ophthalmol Vis Sci. 2007;48:543-549.

24. Upritchard JE, Schuurman CR, Wiersma A, et al. Spread supplemented with moderate doses of vitamin $\mathrm{E}$ and carotenoids re duces lipid peroxidation in healthy, nonsmoking adults. Am J Clin Nutr. 2003;78:985-992

25. Ahsan H, Ali A, Ali R. Oxygen free radicals and systemic autoimmunity. Clin Exp Immunol. 2003;131:398-404.

26. Videla LA, Guerri C. Glutathione and alcohol. In: Vina J, ed. Glutathione: Metabolism and Physiological Functions. Boca Raton, FL: CRC Press; 1990:57-67.

27. Beutler E, Gelbart T. Plasma glutathione in health and in patients with malignant disease. J Lab Clin Med. 1985;105:581-584.

28. Beard KM, Shangari N, Wu B, O'Brien PJ. Metabolism, not autoxidation, plays a role in alpha-oxoaldehyde- and reducing sugarinduced erythrocyte GSH depletion: relevance for diabetes mellitus. Mol Cell Biochem. 2003;252:331-338.

29. Jones DP, Coates RJ, Flagg EW, et al. Glutathione in foods listed in the National Cancer Institute's Health Habits and History Food Frequency Questionnaire. Nutr Cancer. 1992;17:57-75.

30. Anderson ME. Glutathione. In: Punchard NA, Kelly FJ, eds. Free Radicals: A Practical Approach. Oxford, UK: Oxford University Press; 1996:213-226.

31. Anderson ME, Meister A. Dynamic state of glutathione in blood plasma. J Biol Chem. 1980;255:9530-9533.

32. Jones DP, Carlson JL, Samiec PS, et al. Glutathione measurement in human plasma. Evaluation of sample collection, storage and derivatization conditions for analysis of dansyl derivatives by HPLC. Clin Chim Acta. 1998;275:175-184.

33. Griffith OW. Determination of glutathione and glutathione disulfide using glutathione reductase and 2-vinylpyridine. Anal Biochem. 1980;106:207-212.

34. Gherghel D, Griffiths HR, Hilton EJ, Cunliffe IA, Hosking SL Systemic reduction in glutathione levels occurs in patients with primary open-angle glaucoma. Invest Ophthalmol Vis Sci. 2005 46:877-883.

35. van der Veen RL, Berendschot TT, Hendrikse F, Carden D, Makridaki M, Murray IJ. A new desktop instrument for measuring macular pigment optical density based on a novel technique for setting flicker thresholds. Ophthalmic Physiol Opt. 2009;29:127137.

36. Howells $\mathrm{O}$, Eperjesi $\mathrm{F}$, Bartlett $\mathrm{H}$. Measuring macular pigment optical density in vivo: a review of techniques. Graefes Arch Clin Exp Ophthalmol. 2011;249:315-347.

37. Bartlett H, Stainer L, Singh S, Eperjesi F, Howells O. Clinical evaluation of the MPS9000 Macular Pigment Screener. BrJ Ophthalmol. 2010;94:753-756

38. Liang FQ, Godley BF. Oxidative stress-induced mitochondrial DNA damage in human retinal pigment epithelial cells: a possible mechanism for RPE aging and age-related macular degeneration. Exp Eye Res. 2003;76:397-403.

39. Davies NP, Morland AB. Macular pigments: their characteristics and putative role. Prog Retin Eye Res. 2004;23:533-559.

40. Bartlett H, Howells O, Eperjesi F. The role of macular pigment assessment in clinical practice: a review. Clin Exp Optom. 2010; 93:300-308

41. Sternberg Jr P, Davidson PC, Jones DP, Hagen TM, Reed RL, Drews-Botsch C. Protection of retinal pigment epithelium from oxidative injury by glutathione and precursors. Invest Ophthalmol Vis Sci. 1993;34:3661-3668.

42. Yi C, Pan X, Yan H, Guo M, Pierpaoli W. Effects of melatonin in age-related macular degeneration. Ann N Y Acad Sci. 2005;1057: 384-392

43. Reiter RJ, Tan DX, Paredes SD, Fuentes-Broto L. Beneficial effects of melatonin in cardiovascular disease. Ann Med. 2010;42:276285

44. Bendich A. Antioxidant micronutrients and immune responses. Ann N Y Acad Sci. 1990;587:168-180.

45. Pompella A, Visvikis A, Paolicchi A, De Tata V, Casini AF. The changing faces of glutathione, a cellular protagonist. Biochem Pharmacol. 2003;66:1499-1503.

46. Charman WN. Age, lens transmittance, and the possible effects of light on melatonin suppression. Ophthalmic Physiol Opt. 2003; 23:181-187.

47. Skene DJ, Arendt J. Human circadian rhythms: physiological and therapeutic relevance of light and melatonin. Ann Clin Biochem. 2006; $43: 344-353$

48. Turner PL, Mainster MA. Circadian photoreception: ageing and the eye's important role in systemic health. Br J Ophthalmol. 2008;92:1439-1444.

49. Flagg EW, Coates RJ, Jones DP, et al. Plasma total glutathione in humans and its association with demographic and health-related factors. Br J Nutr. 1993;70:797-808.

50. Michelet F, Gueguen R, Leroy P, Wellman M, Nicolas A, Siest G. Blood and plasma glutathione measured in healthy subjects by HPLC: relation to sex, aging, biological variables, and life habits. Clin Chem. 1995;41:1509-1517. 